\title{
Ganho de peso e características da carcaça de cordeiros recebendo diferentes níveis de energia na ração ${ }^{1}$
}

\section{Weight gain and carcass characteristics of lambs receiving different levels of energy in the ration}

\author{
Walter Piola Júnior²; Edson Luis de Azambuja Ribeiro ${ }^{3 *}$ \\ Ivone Yurika Mizubuti ${ }^{4}$; Leandro das Dores Ferreira da Silva ${ }^{5}$; \\ Marco Antonio da Rocha ${ }^{5}$; Marco Aurélio Alves Ferreira Barbosa ${ }^{5}$; \\ Cícero Leandro de Sousa ${ }^{6}$; Fernando Henrique Pereira de Paiva ${ }^{6}$
}

\section{Resumo}

Este trabalho foi realizado com o objetivo de avaliar a influência de diferentes níveis de energia na ração sobre o desempenho e características da carcaça de ovinos terminados em confinamento e abatidos aos 32 quilos de peso vivo. Foram avaliados três tratamentos: 2,23, 2,54 e 2,85 Mcal de EM/kg de MS, sendo o último tratamento, a recomendação estipulada pelo National Research Council - NRC (1985), para cordeiros com ganhos diários de 250 gramas. Foram utilizados 18 cordeiros inteiros, seis por tratamento, mestiços Texel, alimentados com rações isoprotéicas (16,70\% PB). As recomendações de energia propostas pelo NRC (1985) propiciaram ganhos de peso menores do que os esperados. O nível de energia teve efeito linear $(\mathrm{P}<0,05)$ sobre o ganho de peso $(\hat{\mathrm{y}}=-190+141 \mathrm{X})$. Portanto, dietas com maior aporte energético propiciaram maior ganho de peso e consequentemente reduziram o tempo para a terminação dos cordeiros. Carcaças de animais abatidos com pesos similares apresentaram características morfológicas diferentes.

Palavras-chave: Correlação, desempenho, medidas objetivas, produção, ovinos, rendimento

\begin{abstract}
This work was conducted with the objective of evaluating the influence of different levels of energy in the ration on the performance and carcass characteristics of sheep, finished in feedlot and slaughtered with $32 \mathrm{~kg}$ of live weight. Three treatments were evaluated: $2.23,2.54$ and $2.85 \mathrm{Mcal}$ of ME $/ \mathrm{kg}$ of $\mathrm{DM}$, being the last treatment the recommendation of the National Research Council - NRC (1985) for weight daily gains of $250 \mathrm{~g}$ in lambs. Eighteen, intact, Texel crossbred lambs were used; six per treatment. Lambs were fed with isoprotein rations $(16.70 \% \mathrm{CP})$. The energy recommendations proposed by the NRC (1985) resulted in smaller weight gains than expected. The energy level had a linear effect $(\mathrm{P}<0.05)$ on weight gain $(\hat{\mathrm{y}}=-190+141 \mathrm{X})$. Diets with greater energy supply rendered greater weight gains, and consequently less time for the termination of the lambs. Carcasses of animals slaughtered with similar weights presented different morphological characteristics.
\end{abstract}

Key words: Correlation, objective measures, performance, production, sheep, yield

1 Projeto financiado pela Fundação Araucária.

2 Aluno do Programa de Pós-graduação em Ciência Animal da UEL.

3 Professor do Departamento de Zootecnia, CCA, Universidade Estadual de Londrina, Londrina, PR. Bolsista Produtividade do CNPq. E-mail:elar@uel.br.

4 Professor do Departamento de Zootecnia, CCA, Universidade Estadual de Londrina, Londrina, PR, CEP 86051-970. Bolsista Produtividade do CNPq.

5 Professor do Departamento de Zootecnia da UEL.

6 Aluno do Curso de Zootecnia da UEL.

Autor para correspondência 


\section{Introdução}

Apesar de no Brasil a ovinocultura ainda ter importância limitada como atividade produtora, o consumo de carne ovina está crescendo (BRISOLA; SANTO, 2003). Segundo Muller (1993) o baixo consumo de carne ovina observado no país é devido a uma oferta inconstante por parte dos açougues e supermercados, uma má apresentação do produto e excesso de gordura nas carcaças. Dessa forma, a produção de carne ovina vem suprindo apenas uma pequena parte do consumo interno, onde o cordeiro é a categoria mais demandada.

A demanda por cordeiros ocorre porque apresentam um produto final de melhor qualidade, tendo um melhor rendimento de carcaça e eficiência de produção, devido a seu crescimento de forma rápida. Outros fatores, além da idade, também mostram influência no produto final, como a condição sexual (machos inteiros ou castrados), o sexo, o peso ao abate e tipo de alimentação (FIGUEIRÓ; BENAVIDES, 1990).

O confinamento é uma das tecnologias que pode ser empregada para o aumento dos índices de produtividade da ovinocultura e melhoria da qualidade do produto (CARVALHO; SIQUEIRA, 2001). Para se obter uma produção tecnicamente viável é preciso fornecer rações balanceadas, para conseguir maior ganho diário em peso e redução da idade ao abate, com reflexos positivos sobre a qualidade das carcaças e sobre a oferta de carne na entressafra.

Vários fatores podem afetar o rendimento de carcaça, sobretudo a alimentação, que inquestionavelmente, é um dos mais importantes. Dentre os nutrientes a serem supridos, a energia tem recebido atenção especial por ser de fundamental importância para o funcionamento dos órgãos vitais, para a atividade e renovação das células e os processos de utilização dos nutrientes, entre outros (ZUNDT et al., 2001). Ainda, de acordo com Mahgoub, Lu e Early (2000), a suplementação de energia melhora a eficiência de crescimento.
As carcaças são resultados de um processo biológico individual, sobre o qual interferem fatores genéticos, ecológicos e de manejo, diferindo entre si por suas características quantitativas e qualitativas, susceptíveis de identificação (OSÓRIO; OSÓRIO, 2001). O conhecimento e descrição dessas características apresentam uma grande importância tanto para sua comercialização como para sua produção.

O estudo das carcaças é uma avaliação de parâmetros relacionados com medidas objetivas e subjetivas em relação à mesma e deve estar ligado aos aspectos e atributos inerentes à porção comestível. Atualmente, a meta em ovinos de corte é a obtenção de animais capazes de direcionar grande quantidade de nutrientes para a produção de músculos, uma vez que o acúmulo desse tecido é desejável, e reflete a maior parte da porção comestível de uma carcaça (SANTOS; PÉREZ, 2000).

Este trabalho foi realizado com o objetivo de avaliar a influência de diferentes níveis de energia na alimentação, sobre o desempenho e características da carcaça de ovinos terminados em confinamento e abatidos aos 32 quilos de peso vivo.

\section{Material e métodos}

O experimento foi conduzido na Fazenda Escola e Departamento de Zootecnia, do Centro de Ciências Agrárias, da Universidade Estadual de Londrina. O delineamento experimental foi inteiramente casualizado com três tratamentos: 2,23; 2,54 e 2,85 Mcal de EM/kg de MS da ração, e seis repetições. O último tratamento continha o nível de energia estipulado pelo National Research Council - NRC (1985) para cordeiros com ganhos diários de 250 gramas. Os tratamentos com 2,23 e 2,54 Mcal corresponderam, respectivamente, a 78,2 e $89,1 \%$ das recomendações do NRC (1985). Foram usados 18 cordeiros inteiros, mestiços Texel, alimentados com rações isoprotéicas $(16,70 \% \mathrm{~PB})$, duas vezes ao dia (8 e 17 horas). As quantidades fornecidas e sobras foram pesadas diariamente e ajustadas de 
acordo com o consumo dos animais, de maneira a proporcionar sobras diárias de aproximadamente $10 \%$ da quantidade oferecida. As rações foram formuladas com base nos requerimentos de cordeiros em crescimento, segundo o NRC (1985).
A composição percentual e química das rações está contida na Tabela 1. As análises de matéria seca $(\mathrm{MS})$, proteína bruta $(\mathrm{PB})$, extrato etéreo $(\mathrm{EE})$ e fibra em detergente neutro (FDN) foram feitas segundo métodos citados por Silva e Queiroz (2002).

Tabela 1. Composição percentual e química das rações experimentais, com três níveis de energia.

\begin{tabular}{lccc}
\hline & \multicolumn{3}{c}{ Níveis de energia (Mcal de EM/kgMS) } \\
\cline { 2 - 4 } \multicolumn{1}{c}{ Ingredientes } & $\mathbf{2 , 2 3}$ & $\mathbf{2 , 5 4}$ & $\mathbf{2 , 8 5}$ \\
\hline Silagem de Sorgo & \multicolumn{3}{c}{ Composição percentual (\% MS) } \\
Milho & 72,0 & 47,0 & 22,2 \\
Farelo de soja & 4,5 & 29,1 & 53,4 \\
Óleo Soja & 22,0 & 21,2 & 20,5 \\
Sal mineral & 0,0 & 1,2 & 2,4 \\
Uréia & 1,0 & 1,0 & 1,0 \\
& 0,5 & 0,5 & 0,5 \\
\hline \multicolumn{1}{c}{ Nutrientes } & \multicolumn{3}{c}{ Composição química (\% MS) } \\
Matéria seca & 46,4 & 60,8 & 75,1 \\
Nutrientes digestíveis totais & 61,7 & 70,4 & 79,0 \\
Proténa bruta & 16,7 & 16,7 & 16,7 \\
Extrato etéreo & 1,9 & 3,4 & 5,0 \\
Fibra em detergente neutro & 46,8 & 34,5 & 22,3 \\
\hline
\end{tabular}

No inicio do experimento os cordeiros tinham idade média de $70 \pm 9$ dias e peso inicial $(\mathrm{PI})$ médio de $20 \pm 2,7 \mathrm{~kg}$ e passaram por um período de adaptação de 15 dias. No primeiro dia da adaptação os animais receberam vermífugo de amplo espectro, sendo então confinados em baias individuais em aprisco coberto e com piso ripado e elevado do solo.

Os animais foram pesados a cada sete dias para se estabelecer o ganho médio diário, após jejum prévio de alimentos sólidos de 18 horas. Os cordeiros foram abatidos conforme atingiam peso vivo médio de 32 $\mathrm{kg}$, portanto, com diferentes idades. As medidas nas carcaças foram feitas de acordo com Silva Sobrinho (2001) e Osório e Osório (2005). Imediatamente após o abate o conteúdo gastrintestinal foi pesado para a obtenção do peso de corpo vazio (PCVZ), onde $\mathrm{PCVZ}=$ peso ao abate - peso do conteúdo gastrintestinal.
As carcaças foram pesadas imediatamente após o abate (PCQ = peso de carcaça quente) e após refrigeração $(\mathrm{PCF}=$ peso de carcaça fria $)$ a $2^{\circ} \mathrm{C}$ por 24 horas em câmara fria. Em seguida, foram registradas as medidas objetivas das carcaças. Foi avaliado o comprimento interno da carcaça (distância máxima entre o bordo anterior da sínfise ísquio-pubiana e o bordo anterior da primeira costela, em seu ponto médio), a profundidade do tórax (distância máxima entre o esterno e o dorso da paleta), o comprimento do braço (distância entre o olecrano e a borda anterior da superfície carpo metacarpiana), a espessura de coxão, aferida com um compasso, colocado nas bordas mais proeminentes do coxão, onde a distância das duas extremidades desse compasso foi medida com fita métrica, e perímetro de braço, medido com fita métrica envolvendo o braço à metade de seu comprimento. Foi avaliada, também, a perda de peso por resfriamento (PCQ - PCF) x 100) / PCQ), o rendimento biológico ou verdadeiro (PCQ / PCVZ) 
x 100), o rendimento de carcaça quente (PCQ / peso ao abate) x 100), o rendimento de carcaça fria (PCF / peso ao abate) x 100), e a compacidade da carcaça (PCF / comprimento da carcaça).

Após as mensurações as carcaças foram divididas longitudinalmente, na altura da linha média, em duas partes, sendo a parte esquerda seccionada em cinco regiões anatômicas. Tais regiões compreenderam: Perna: base óssea que abrange a região do ilíaco (ílio), ísquio, púbis, vértebras sacrais, as duas primeiras vértebras coccígeas, fêmur, tíbia e tarso obtido por corte perpendicular à coluna entre a última vértebra lombar e a primeira sacra; Lombo: compreende a região das vértebras lombares, obtido perpendicularmente à coluna, entre a $13^{\mathrm{a}}$ vértebra dorsal-primeira lombar e última lombar-primeira sacra; Costelas: compreende as 13 vértebras torácicas, com as costelas correspondentes ao esterno; Paleta: região que compreende a escápula, úmero, rádio, ulna e o carpo; Pescoço: refere-se às sete vértebras cervicais, obtidas por corte oblíquo entre a sétima cervical e a primeira torácica.

As análises estatísticas foram efetuadas usandose o pacote estatístico Statistical Analysis System Institute - SAS (1994). Os dados foram submetidos à análise de variância, com modelo matemático incluindo o efeito fixo de tratamento (nível de energia) e análise de regressão, em função dos três níveis de energia. Foi efetuada, também, a correlação de Pearson entre as variáveis estudadas. Para tal, inicialmente realizou-se as análises de correlação separadas para cada tratamento, porém, como os resultados foram praticamente idênticos, resolveuse agrupar todos os dados em uma única análise.

\section{Resultados e discussão}

O ganho médio diário (GMD), dias no confinamento (DIAS) e perda por resfriamento foram afetadas significativamente $(\mathrm{P}<0,05)$ pelos tratamentos (Tabelas 2 e 3 ). Estes resultados demonstram que o nível energético mais elevado, diminuiu o tempo até o abate dos animais, por apresentarem os maiores ganhos diários. A perda por resfriamento foi menor nos tratamentos com maior nível energético, concordando com os resultados observados por Garcia, Monteiro e Costa (2003), que utilizaram cordeiros em crescimento com diferentes níveis de energia, alimentados e terminados em creep feeding. As perdas por resfriamento são menores que às observadas por Mendonça et al. (2003), que citaram valores de 3,77 e 5,94\%, respectivamente, para carcaças de borregos Ideal e Corriedale, abatidos com um ano de idade. Os autores comentaram que diferenças nas perdas por resfriamento podem ser influenciadas pelo genótipo dos animais, e principalmente pela distribuição da gordura de cobertura.

Tabela 2. Médias para pesos inicial (PVI), ao abate (PVA) e corpo vazio (PCVZ), ganho de peso médio diário (GMD) e dias no confinamento (DIAS) de cordeiros alimentados com três níveis de energia.

\begin{tabular}{|c|c|c|c|c|c|c|}
\hline \multicolumn{7}{|c|}{ Nível de energia (Mcal/kgMS) } \\
\hline Variável & 2,23 & 2,54 & 2,85 & Regressão & $\mathrm{R}^{2}$ & CV (\%) \\
\hline PVI, kg & 20,4 & 19,9 & 20,3 & $\tilde{y}=20,2$ & - & 13,7 \\
\hline PVA, kg & 32,7 & 32,8 & 31,4 & $\tilde{y}=32,3$ & - & 11,9 \\
\hline PCVZ, kg & 27,1 & 28,0 & 27,2 & $\tilde{y}=27,4$ & - & 12,5 \\
\hline GMD, g & $120,6^{\mathrm{b}}$ & $179,8^{\mathrm{a}}$ & $208,2^{\mathrm{a}}$ & $\hat{y}=-190+141 X$ & 0,6 & 17,4 \\
\hline DIAS & $102^{\mathrm{a}}$ & $72^{\mathrm{b}}$ & $53^{c}$ & $\hat{\mathrm{y}}=276,4086-79,0323 \mathrm{X}$ & 1,0 & 3,6 \\
\hline
\end{tabular}

a,b,c Médias seguidas de letras diferentes, na linha, diferem pelo teste t a $5 \%$ de probabilidade. $\mathrm{R}^{2}=$ coeficiente de determinação, $\mathrm{CV}=$ coeficiente de variação. 
Tabela 3. Médias para pesos de carcaça quente (PCQ) e fria (PCF), perdas no resfriamento (PR), e rendimentos verdadeiro $(\mathrm{RV})$, carcaça quente (RCQ) e fria $(\mathrm{RCF})$ de cordeiros alimentados com três níveis de energia.

\begin{tabular}{|c|c|c|c|c|c|c|}
\hline \multicolumn{7}{|c|}{ Nível de energia (Mcal/kgMS) } \\
\hline Variável & 2,23 & 2,54 & 2,85 & Regressão & $\mathrm{R}^{2}$ & CV $(\%)$ \\
\hline PCQ, kg & 15,1 & 14,9 & 14,9 & $\tilde{y}=15,0$ & - & 15,6 \\
\hline PCF, kg & 14,6 & 14,3 & 14,5 & $\tilde{y}=14,5$ & - & 15,9 \\
\hline $\mathrm{PR}, \%$ & $3,4^{\mathrm{ab}}$ & $3,7^{\mathrm{a}}$ & $2,6^{\mathrm{b}}$ & $\hat{y}=-52,148+45,459 X-9,206 X^{2}$ & 0,35 & 24,0 \\
\hline $\mathrm{RV}, \%$ & 55,6 & 53,0 & 54,6 & $\tilde{y}=54,4$ & - & 4,8 \\
\hline $\mathrm{RCQ}, \%$ & 46,0 & 45,1 & 47,4 & $\tilde{y}=46,2$ & - & 5,9 \\
\hline $\mathrm{RCF}, \%$ & 44,4 & 43,4 & 46,1 & $\tilde{y}=44,7$ & - & 6,4 \\
\hline
\end{tabular}

${ }^{\mathrm{a}, \mathrm{b}}$ Médias seguidas de letras diferentes, na linha, diferem pelo teste $\mathrm{t}$ a $5 \%$ de probabilidade.

$\mathrm{R}^{2}=$ coeficiente de determinação, $\mathrm{CV}=$ coeficiente de variação.

O tempo médio observado para os animais atingirem peso vivo ao abate de $32 \mathrm{~kg}$ demonstra a influência positiva, do nível de energia na dieta, em diminuir o tempo de confinamento, semelhante ao observado por Alves et al. (2003). Os dados reforçam o exposto por Cunha et al. (2001) e Pinto et al. (2005), os quais observaram que níveis maiores de energia possibilitam menores idades ao abate.

Os valores encontrados para ganho de peso tiveram efeito linear crescente, e foram similar aos obtidos por Alves et al. (2003) e superiores aos encontrados por Pinto et al. (2005), que trabalharam com cordeiros em crescimento recebendo diferentes fontes de volumosos em confinamento. Por outro lado, foram inferiores aos observados por Garcia et al. (2000), que utilizaram cordeiros em confinamento recebendo diferentes níveis energéticos em creep feeding.

Apesar de ter havido efeito significativo $(\mathrm{P}<0,05)$ dos teores de energia da dieta, sobre o ganho de peso (Tabela 2), os mesmos foram inferiores ao estabelecido pelo NRC (1985). Resultados semelhantes foram observados por diferentes autores. Fernandes et al. (1996), analisando o efeito de dois planos nutricionais sobre o desempenho de cordeiros F1 Santa Inês x Crioula, em confinamento, cujas rações continham 2,54 e 2,62 Mcal de EM/kg de MS, e formuladas para ganho médio diário de 0,200 e $0,250 \mathrm{~kg} / \mathrm{dia}$, respectivamente, obtiveram média para ganho de peso diário de 0,128 e 0,156 $\mathrm{kg} /$ dia. Garcia et al. (2000), avaliando dietas que continham 2,47; 2,38 e 2,39 $\mathrm{kcal}$ de EM/kg de MS, encontraram valores para ganho de peso de 0,211; 0,193 e 0,195 kg/dia, respectivamente. Em trabalho semelhante, Mahgoub, Lu e Early (2000) avaliaram dietas contendo 2,4; 2,5; 2,7 Mcal EM/ $\mathrm{kg}$ de MS para ovinos em crescimento e obtiveram ganhos de peso de 0,090; 0,115; e 0,147 kg/dia, respectivamente. Martins et al. (1999), quando avaliaram o desempenho de cordeiros mestiços Texel em confinamento, alimentados com dois níveis de energia na dieta $(2,17$ e 2,72 Mcal EM/kg de MS) obtiveram ganho de peso diário de 0,116 e $0,159 \mathrm{~kg} / \mathrm{dia}$, respectivamente.

Estes resultados demonstram que as indicações de ganho de peso das tabelas de exigências nutricionais podem não ser alcançadas nas nossas condições, pois vários fatores podem influenciar, positiva ou negativamente, os resultados, tais como: diferenças de potencial genético dos animais, diferenças nas dietas utilizadas, além de outros fatores ambientais (temperatura, umidade relativa do ar, luminosidade, entre outros).

Os pesos inicial e ao abate, de corpo vazio e de carcaça quente e fria (Tabelas 2 e 3), não apresentaram diferenças, provavelmente por se tratarem de animais de pesos médios padronizados, $20 \mathrm{~kg}$ inicial e $32 \mathrm{~kg}$ final.

Os resultados encontrados neste trabalho estão de acordo com diversos trabalhos realizados 
com ovinos, em que o peso de carcaça fria de cordeiros abatidos com 20,25 a 35,07 kg de peso corporal variou de 7,90 a $15,55 \mathrm{~kg}$ e o rendimento de carcaça fria, de 38,86 a 47,97\% (COSTAÑER; ASTIZ; ALFRANCA, 1992; OSÓRIO et al., 1996, 1999; PIRES et al., 1999; SILVA; SALVADO; PORTUGAL, 1994).

$\mathrm{Na}$ tabela 4, onde se encontram os resultados das medidas objetivas das carcaças, observa-se que houve efeito significativo dos tratamentos $(\mathrm{P}<0,05)$, sobre o comprimento interno de carcaça e espessura de coxão. Os valores para comprimento interno de carcaça, de forma geral, se assemelham aos observados por Garcia, Monteiro e Costa (2003) em cordeiros recebendo diferentes níveis de energia em creep feeding. Porém, no presente trabalho, as carcaças dos animais do tratamento com maior nível de energia, apresentaram valores menores do que as carcaças dos animais que tiveram níveis menores de energia. Uma possível explicação para este fato é que os animais alimentados com menor nível energético foram abatidos mais tarde, permitindo um maior crescimento ósseo, apesar dos pesos ao abate serem semelhantes.

Tabela 4. Médias para comprimento interno de carcaça (CIC), profundidade de tórax (PT), comprimento de braço (CB), espessura de coxão (EC), perímetro de braço (PB) e compacidade de carcaça (CC) de cordeiros alimentados com três níveis de energia.

\begin{tabular}{lcccccc}
\hline \multicolumn{7}{c}{ Nível de energia (Mcal/kgMS) } \\
\hline Variável & 2,23 & 2,54 & 2,85 & Regressão & $\mathrm{R}^{2}$ & CV $(\%)$ \\
\hline CIC, cm & $58,3^{\mathrm{a}}$ & $58,4^{\mathrm{a}}$ & $54,8^{\mathrm{b}}$ & $\hat{\mathrm{y}}=71,533-5,645 \mathrm{X}$ & 0,3 & 3,5 \\
PT, cm & 26,3 & 25,6 & 24,5 & $\tilde{y}=25,4$ & - & 6,4 \\
$\mathrm{CB}, \mathrm{cm}$ & 18,8 & 18,3 & 17,8 & $\tilde{y}=18,3$ & - & 5,8 \\
$\mathrm{EC}, \mathrm{cm}$ & $7,8^{\mathrm{b}}$ & $9,1^{\mathrm{a}}$ & $8,7^{\mathrm{ab}}$ & $\hat{\mathrm{y}}=-40,034+37,106 \mathrm{X}-7,023 \mathrm{X}^{2}$ & 0,3 & 9,5 \\
$\mathrm{~PB}, \mathrm{~cm}$ & 14,6 & 15,3 & 14,8 & $\tilde{y}=14,9$ & - & 6,4 \\
$\mathrm{CC}, \mathrm{kg} / \mathrm{cm}$ & 0,25 & 0,24 & 0,26 & $\tilde{y}=0,25$ & - & 15,0 \\
\hline
\end{tabular}

${ }^{a, b}$ Médias seguidas de letras diferentes na mesma linha diferem pelo teste t a $5 \%$ de probabilidade, $\mathrm{R}^{2}=$ coeficiente de determinação, $\mathrm{CV}=$ coeficiente de variação

Para a espessura de coxão os valores diferem daqueles divulgados por Bueno, Cunha e Santos (2000), onde os valores aumentaram com o decorrer da idade. Neste trabalho houve diferença devido aos níveis de energia, onde a espessura tendeu a aumentar e depois diminuir, com o aumento do nível de energia. A espessura de coxão correlaciona-se com musculosidade da carcaça, principalmente dos cortes do pernil. Os animais que receberam o maior nível energético apresentaram carcaças mais curtas a um mesmo peso, consequentemente apresentaram maior musculosidade.

Pires, Carneiro e Muller (2006) avaliaram cordeiros desmamados e não desmamados e não encontraram diferenças para as características das carcaças. Segundo os autores, isto ocorreu porque o peso de abate foi o mesmo para todos os tratamentos.
Motta (2000) relatou que cordeiros abatidos com maiores pesos possuem maior espessura de coxão, e que o peso ao abate, influência no comprimento de carcaça. O índice de compacidade de carcaça, não foi influenciado pela inclusão de energia na dieta, corroborando com Martins et al. (1999), quando trabalharam com 2,17 e 1,72 Mcal EM/kg de MS.

$\mathrm{Na}$ Tabela 5 são apresentadas às correlações fenotípicas entre as variáveis estudadas. Observouse que as maiores correlações foram entre pesos de carcaça fria e quente e rendimentos de carcaça quente e fria. Observou-se, também, que apesar dos pesos vivos ao abate estarem altamente correlacionados com os pesos de carcaça, não foram significativamente correlacionados com os rendimentos de carcaça quente ou fria. 


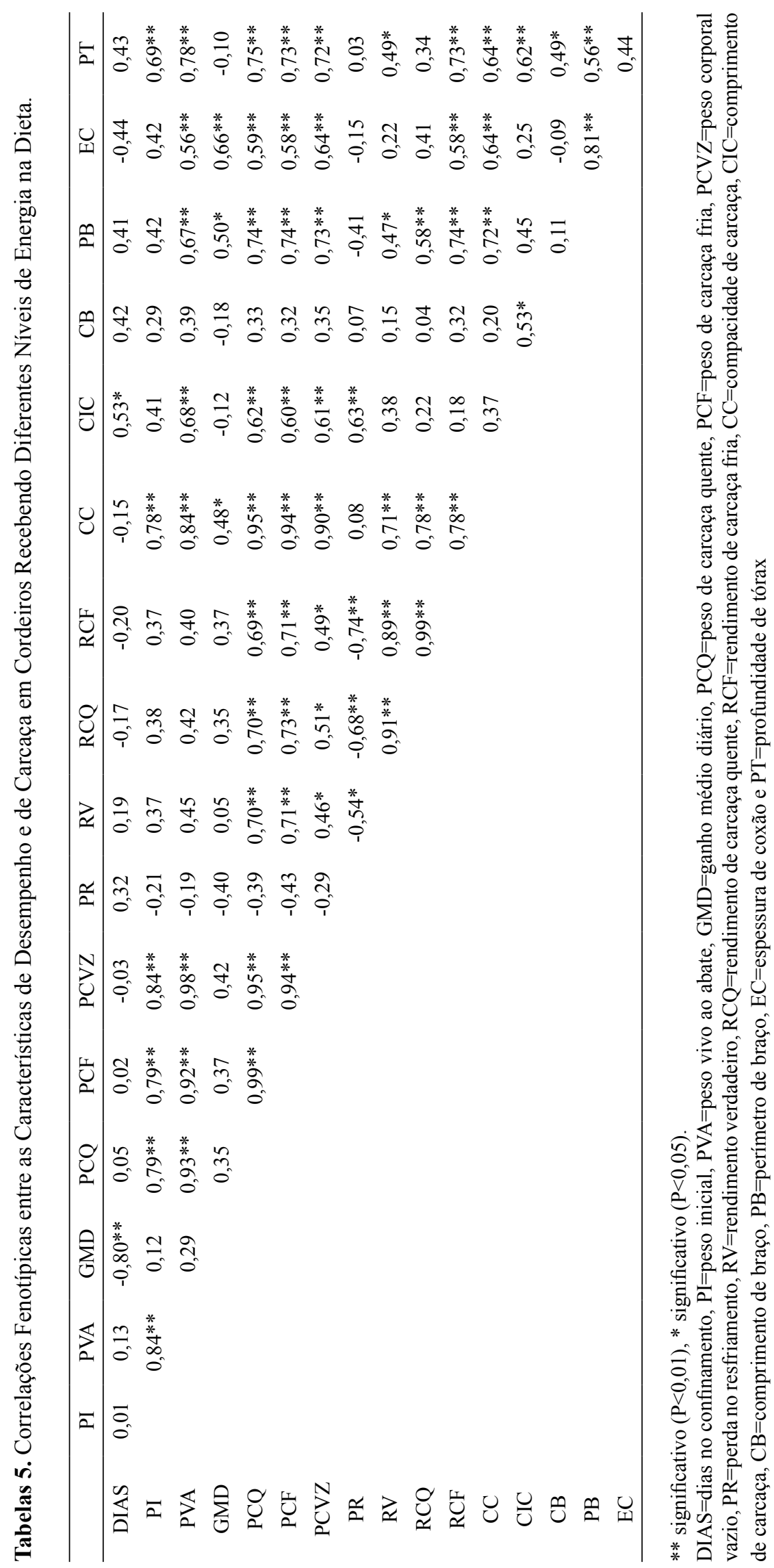


Animais mais pesados no início do experimento, também foram os mais pesados ao abate, porém o peso inicial não esteve correlacionado com o ganho médio diário.

Um resultado bastante interessante é o que mostra que a compacidade de carcaça esteve correlacionada positivamente com as principais características produtivas, tais como, peso ao abate e de carcaça e com o rendimento de carcaça, além de perímetro de braço, espessura de coxão e profundidade de tórax. Este resultado demonstrou que animais com maior massa muscular são mais eficientes na produção de carcaça. Comprimento de carcaça apresentou correlação positiva com os pesos de abate e de carcaça.

\section{Conclusões}

Dietas com maior aporte energético propiciam maiores ganhos de peso e consequentemente menor tempo para a terminação dos cordeiros, além de menor perda de peso das carcaças no resfriamento.

Carcaças de animais abatidos com pesos similares podem apresentar características morfológicas diferentes.

\section{Referências}

ALVES, K. S.; CARVALHO, F. F. R.; FERREIRA, M. A.; VÉRAS, A. S. C. Níveis de energia em dietas para ovinos Santa Inês: Características de carcaça e constituintes corporais. Revista Brasileira de Zootecnia, Viçosa, v. 32, n. 6, Supl. 2, p. 1927-1936, 2003.

BRISOLA, M. V.; SANTO, E. E. Panorama da cadeia produtiva da ovinocultura no Brasil. In: SIMPÓSIO MINEIRO DE OVINOCULTURA, 3., Lavras, 2003. Anais... Lavras: UFLA, 2003. p. 11-24.

BUENO, M. S.; CUNHA, E. A.; SANTOS, L. E. Características de carcaça de cordeiros Suffolk abatidos em diferentes idades. Revista Brasileira de Zootecnia, Viçosa, v. 29, n. 6, p. 1803-1810, 2000.

CARVALHO, S. R. S. T.; SIQUEIRA, E. R. Produção de cordeiros em confinamento. In: SIMPÓSIO MINEIRO
DE OVINOCULTURA: PRODUÇÃO DE CARNE NO CONTEXTO ATUAL, 1., Lavras, 2001. Anais... Lavras: UFLA, 2001. p. 21-48.

COSTAÑER, J. L. O.; ASTIZ, C. S.; ALFRANCA, I. S. Produccion de carne en la agrupacion ovina Churra Tensina: calidad de la canal y de la carne en los tipos ternasco y cordero de cebo. Archivos de Zootecnia, Cordoba, v. 41, n. 153, p. 197-208, 1992.

CUNHA, E. A.; BUENO, M. S.; SANTOS, L. E.; RODA, D. S.; OTSUK, I. P. Desempenho e características de carcaça de cordeiros Suffolk alimentados com diferentes volumosos. Ciência Rural, Santa Maria, v. 31, n. 4, p. 671-676, 2001.

FERNANDES, F. D.; BARROS, N. N.; ARAUJO, M. R. A.; FIGUEIREDO, E. A. P. Efeito de dois planos nutricionais sobre o desempenho de cordeiros F1 Santa Inês x Crioula em confinamento. In: EMPRESA BRASILEIRA DE PESQUISA AGROPECUÁRIA EMBRAPA. Relatório técnico do Centro Nacional de Pesquisa de Caprinos, 1987-1995. Sobral: EMBRAPACNPC, 1996. p. 69-72.

FIGUEIRÓ, P. R. P.; BENAVIDES, M. V. Produção de carne ovina. In: REUNIÃO ANUAL DA SOCIEDADE BRASILEIRA DE ZOOTECNIA, 27., Campinas, 1990. Anais... Campinas: Unicamp, 1990. p. 15-31.

GARCIA, I. F. F.; PÉREZ, J. R. O.; TEIXEIRA, J. C.; BARBOSA, C. M. P. Desempenho de cordeiros Texel x Bergamácia, Texel x Santa Inês e Santa Inês puros terminados em confinamento, alimentados com casca de café como parte da dieta. Revista Brasileira de Zootecnia, Viçosa, v. 29, n. 2, p. 564-572, 2000.

GARCIA, C. A.; MONTEIRO, A. L. G.; COSTA, C. Medidas Objetivas e Composição Tecidual da Carcaça de Cordeiros Alimentados com Diferentes Níveis de Energia em Creep Feeding. Revista Brasileira de Zootecnia, Viçosa, v. 32, n. 6, p. 1380-1390, 2003.

MAHGOUB, O.; LU, C. D.; EARLY, R. J. Effects of dietary energy density on feed intake, body weiht gain and carcass chemical composition of Omani growing lambs. Small Ruminant Research, Amsterdam, v. 37, n. 1, p. 35-42, 2000.

MARTINS, E. N.; MACEDO, F. A. F.; MACEDO, R. M. G.; MAESTA, S. A. Desempenho e características quantitativas da carcaça de cordeiros mestiços Texel, terminados em confinamento, com diferentes níveis de energia. In: REUNIÃO ANUAL DA SOCIEDADE BRASILEIRA DE ZOOTECNIA, 36., 1999, Porto Alegre. Anais... Porto Alegre: SBZ, 1999. p. 347. 
MENDONÇA, G.; OSÓRIO, J. C.; OLIVEIRA, N. M.; OSÓRIO, M. T.; ESTEVES, R.; WIENGARD, M. M. Morfologia, características da carcaça e componentes do peso vivo em borregos Corriedale e Ideal. Ciência Rural, Santa Maria, v. 33, n. 2, p. 351-355, 2003.

MOTTA, O. S. Ganho de peso, características da carcaça de cordeiros em diferentes métodos de alimentação, pesos de abate e produção e leite de ovelhas. 2000. Dissertação (Mestrado em Zootecnia) - Universidade Federal de Santa Maria, Santa Maria.

MÜLLER, L. Qualidade da carne - tipificação de caraças bovinas e ovinas. In: SIMPÓSIO REUNIÀO ANUAL DA SOCIEDADE BRASILEIRA DE ZOOTECNIA, 30., 1993, Rio de Janeiro. Anais... Viçosa: SBZ, 1993. p. 53-69.

NATIONAL RESEARCH COUNCIL - NRC. Nutrient Requirements of Sheep. 6. ed. Washington: National Academy Press, 1985.

OSÓRIO, J. C.; OLIVEIRA, N. M.; NUNES, A. P.; POUEY, J. L. Produção de carne em ovinos de cinco genótipos. 3. Perdas e morfologia. Ciência Rural, Santa Maria, v. 26, n. 3, p. 447-481, 1996.

OSÓRIO, J. C.; JARDIM, P. O. C.; PIMENTEL, M. A.; POUEY, J.; OSÓRIO, M. T. M.; LUDER, W. E.; BORBA, M. F. Produção de carne entre cordeiros castrados e não castrados. 1 - Cruzas Hampshire Down x Corriedale. Ciência Rural, Santa Maria, v. 29, n. 1, p. 135-138, 1999.

OSÓRIO, J. C. S.; OSÓRIO, M. T. M. Produção de carne ovina: Técnicas de avaliação "in vivo" e na carcaça. 2. ed. Pelotas: Editora Universitária, 2005.

. Sistemas de avaliação de carcaças no Brasil. In: SIMPÓSIO MINEIRO DE OVINOCULTURA, 1., 2001, Lavras, MG. Anais... Lavras: UFLA, 2001. p. 157-196.

PINTO, C. W. C.; SOUZA, W. H.; PIMENTA FILHO, E. C.; CUNHA, M. G. G.; GONZAGA NETO, S. Desempenho de cordeiros Santa Inês terminados com diferentes fontes de volumosos em confinamento. Revista Agropecuária Técnica, Areia, v. 26, n. 2, p. 123-128, 2005.

PIRES, C. C.; ARAÚJO, J. R.; BERNARDES. R. A. C.; LANES, R. C.; JUNGES, E. R. V. Desempenho e características da carcaça de cordeiros de três grupos genéticos abatidos ao mesmo estágio de maturidade. Ciência Rural, Santa Maria, v. 29, n. 1, p. 155-158, 1999.

PIRES, C. C.; CARNEIRO, R. M.; MÜLLER, L. Avaliação da carcaça e componentes do peso vivo, de cordeiros de parto simples desmamados, parto simples não desmamados. Revista Brasileira de Agrociência, Pelotas, v. 12, n. 1, p. 93-97, 2006.

SANTOS, C. L.; PÉREZ, J. R. O. Cortes comerciais de cordeiros Santa Inês. In: ENCONTRO MINEIRO DE OVINOCULTURA, 1., 2000, Lavras. Anais... Lavras: Ed. UFLA, 2000. p. 149-168.

SILVA, J. S.; SALVADO, A. L.; PORTUGAL, A. V. Estudo do crescimento e da composição das carcaças de borregos da raça Churra da terra quente. Revista Portuguesa de Zootecnia, Vila Real, v. 1, n. 2, p. 127136, 1994.

SILVA SOBRINHO, A. G. Criação de ovinos. Jaboticabal: FUNEP, 2001.

SILVA, D. J.; QUEIROZ, C. Análise de alimentos: métodos químicos e biológicos. Viçosa: Ed. UFV, 2002.

STATISTICAL ANALYSIS SYSTEM INSTITUTE - SAS. SAS/STAT User's Guide. Cary: SAS Institute, 1994.

ZUNDT, M.; MACEDO, F. A. F.; ALCADE, C. R.; MEXIA, A. A.; MARTINS, E. N.; PERUZI, A. Z.; SANTOS, V. C. Características de carcaça de caprinos alimentados com diferentes níveis energéticos In: REUNIÃO ANUAL DA SOCIEDADE BRASILEIRA DE ZOOTECNIA, 38., 2001, Piracicaba. Anais... Piracicaba: Sociedade Brasileira de Zootecnia, 2001. p. 992. 
